\title{
Analgesic Effects of Repetitive Transcranial Magnetic Stimulation at Different Stimulus Parameters for Neuropathic Pain: A Randomized Study
}

\author{
Nobuhiko Mori, PT, MHSc ${ }^{1,2}{ }^{\circ}$; Koichi Hosomi, MD, PhD ${ }^{1,2} \oplus_{\text {; }}$ \\ Asaya Nishi, MD²; Satoru Oshino, MD, PhD²; Haruhiko Kishima, MD, PhD²; \\ Youichi Saitoh, MD, PhD ${ }^{1,2}$
}

\begin{abstract}
Objectives: The aim of the present study was to investigate the analgesic effects of repetitive transcranial magnetic stimulation over the primary motor cortex (M1-rTMS) using different stimulation parameters to explore the optimal stimulus condition for treating neuropathic pain.

Materials and Methods: We conducted a randomized, blinded, crossover exploratory study. Four single sessions of M1-rTMS at different parameters were administered in random order. The tested stimulation conditions were as follows: $5-\mathrm{Hz}$ with 500 pulses per session, 10- $\mathrm{Hz}$ with 500 pulses per session, 10- $\mathrm{Hz}$ with 2000 pulses per session, and sham stimulation. Analgesic effects were assessed by determining the visual analog scale (VAS) pain intensity score and Short-Form McGill Pain Questionnaire 2 (SF-MPQ2) score immediately before and immediately after intervention.

Results: We enrolled 22 adults (age: $59.8 \pm 12.1$ years) with intractable neuropathic pain. Linear-effects models showed significant effects of the stimulation condition on changes in VAS pain intensity $(p=0.03)$ and SF-MPQ2 $(p=0.01)$. Tukey multiple comparison tests revealed that $10-\mathrm{Hz}$ rTMS with 2000 pulses provided better pain relief than sham stimulation, with greater decreases in VAS pain intensity $(p=0.03)$ and SF-MPQ2 $(p=0.02)$.

Conclusions: The results of this study suggest that high-dose stimulation (specifically, 10-Hz rTMS at 2000 pulses) is more effective than lower-dose stimulation for treating neuropathic pain.

Keywords: Crossover exploratory study, intractable neuropathic pain, motor cortex stimulation, optimal stimulation condition, repetitive transcranial magnetic stimulation

Conflict of Interest: Nobuhiko Mori, Koichi Hosomi, and Youichi Saitoh are members of the Department of Neuromodulation and Neurosurgery, Osaka University Graduate School of Medicine, which is a joint research department established with sponsorship from Teijin Pharma Limited. Dr. Mori reports grants from JSPS during the conduct of the study. Dr. Hosomi reports grants from AMED and JSPS during the conduct of this study. The remaining authors have no conflicts of interest to disclose.
\end{abstract}

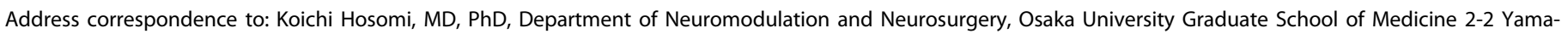
daoka, Suita, Osaka 565-0871, Japan. Email: k-hosomi@nsurg.med.osaka-u.ac.jp

\footnotetext{
1 Department of Neuromodulation and Neurosurgery, Osaka University Graduate School of Medicine, Osaka, 565-0871, Japan; and

2 Department of Neurosurgery, Osaka University Graduate School of Medicine, Osaka, 565-0871, Japan
}

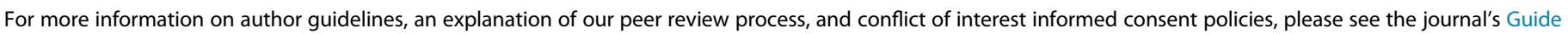
for Authors.

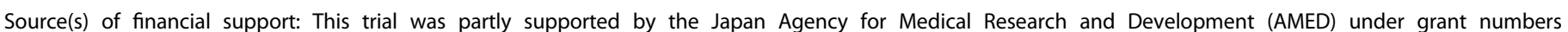

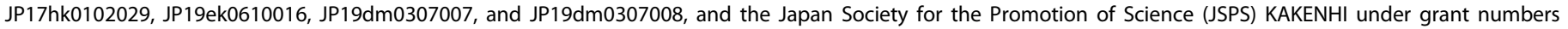

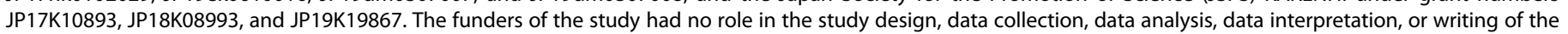
report.

[Correction added on 28 January 2021, after first online publication: the order of authors has been updated.] 


\section{INTRODUCTION}

Neuropathic pain is defined as pain caused by a lesion or disease of the somatosensory nervous system, such as a traumatic nerve injury, stroke, or spinal cord injury. ${ }^{1}$ Neuropathic pain is a refractory chronic pain condition ${ }^{2,3}$ with an estimated prevalence of $3 \%-17 \%$ of the general population. ${ }^{4,5}$ Neuropathic pain may not only reduce a person's activities of daily living and quality of life (QOL), but it may also impose substantial economic burdens on individuals and society. ${ }^{6,7}$ Consistent with the refractory nature of neuropathic pain, the latest comprehensive algorithm includes up to six levels of treatment. ${ }^{8}$ There is a clear need for the development of novel noninvasive therapeutic methods for intractable neuropathic pain.

Stimulation of the primary motor cortex (M1) using repetitive transcranial magnetic stimulation (rTMS) is a noninvasive, safe method of stimulating the brain, $^{9-11}$ and several studies have investigated the analgesic effects of rTMS for various refractory disorders using different stimulation intensities, frequencies, number of pulses, and number of sessions. ${ }^{12-18}$ We previously reported that M1 was a more effective stimulation target than other cortices, $^{19}$ high-frequency (5- or $10-\mathrm{Hz}$ ) rTMS was more effective than low-frequency $(1-\mathrm{Hz}) \mathrm{rTMS}^{14}$ and the effects of a single session of rTMS were short-lasting. ${ }^{20}$ Moreover, in our pilot randomized, blinded, controlled, crossover trial of patients with neuropathic pain in seven centers in Japan, ten daily rTMS treatments produced transient, modest pain relief. ${ }^{21}$ Recent meta-analyses and therapeutic guidelines reported that highfrequency $(\geq 5 \mathrm{~Hz})$ rTMS of M1 was safe and resulted in transient pain-relieving effects for neuropathic pain. ${ }^{9,22}$

Based on the promising earlier results, we subsequently conducted a large rigorous randomized, blinded, controlled, parallel trial involving 144 patients with neuropathic pain. The results showed that five daily sessions of rTMS over M1 with 500 pulses/ session at $5 \mathrm{~Hz}$ did not achieve better pain relief than sham stimulation. $^{23}$ In addition, when we compared $5-\mathrm{Hz}$ rTMS with 500 pulses vs 1500 pulses in patients with neuropathic pain, increasing the number of pulses did not improve pain relief. ${ }^{24}$ One potential reason for these negative results was suboptimal stimulus conditions. The majority of recent studies reporting positive results used higher doses than our previous trials, such as $10-20 \mathrm{~Hz}$ and 2000-3000 pulses/session. ${ }^{25-28}$ Although the optimal stimulation frequency and number of pulses per session have not yet been established, it is likely that doses higher than 500 pulses/session at $5 \mathrm{~Hz}$ are more effective for treating pain. Furthermore, no published studies have directly compared the analgesic effectiveness of different high-frequency stimulations and stimulus pulses for neuropathic pain. Therefore, we conducted a study to explore the optimal stimulus conditions for treating neuropathic pain by comparing the analgesic effects of rTMS over the M1 hand area contralateral to the painful side using different stimulation parameters.

\section{MATERIALS AND METHODS}

\section{Study Design}

We performed a randomized, single-blinded, sham-controlled, crossover exploratory study at Osaka University Hospital from April 2017 through October 2018. The study was conducted in accordance with the Declaration of Helsinki and Japanese ethical guidelines for clinical studies. The study's protocol was approved by the Ethics Committee of Osaka University Hospital (approval number: 16309), and written informed consent was obtained from all patients participating in the study.

The participants were recruited from the outpatient clinic of the Department of Neurosurgery, Osaka University Hospital. Each patient underwent four rTMS sessions (three different active stimulations and one sham stimulation) in a crossover manner, with at least two weeks between each session (Fig. 1). The order of stimulation conditions was allocated using a computer-generated simple randomization method. The patients were identified by sequential numbers assigned at randomization. The patients and assessors were blinded to the intervention throughout the study.

\section{Patients}

Patients with neuropathic pain (based on the grading system of the International Association for the Study of Pain [IASP $]^{1,29,30}$ ) were enrolled in this study. All participants met these inclusion criteria: 1) intractable pain for six months or longer, 2) 30-mm or higher visual analog scale (VAS) pain intensity at baseline (scale range: 0-100 mm), 3) insufficient pain relief despite receiving drugs for neuropathic pain or a history of receiving drugs for neuropathic

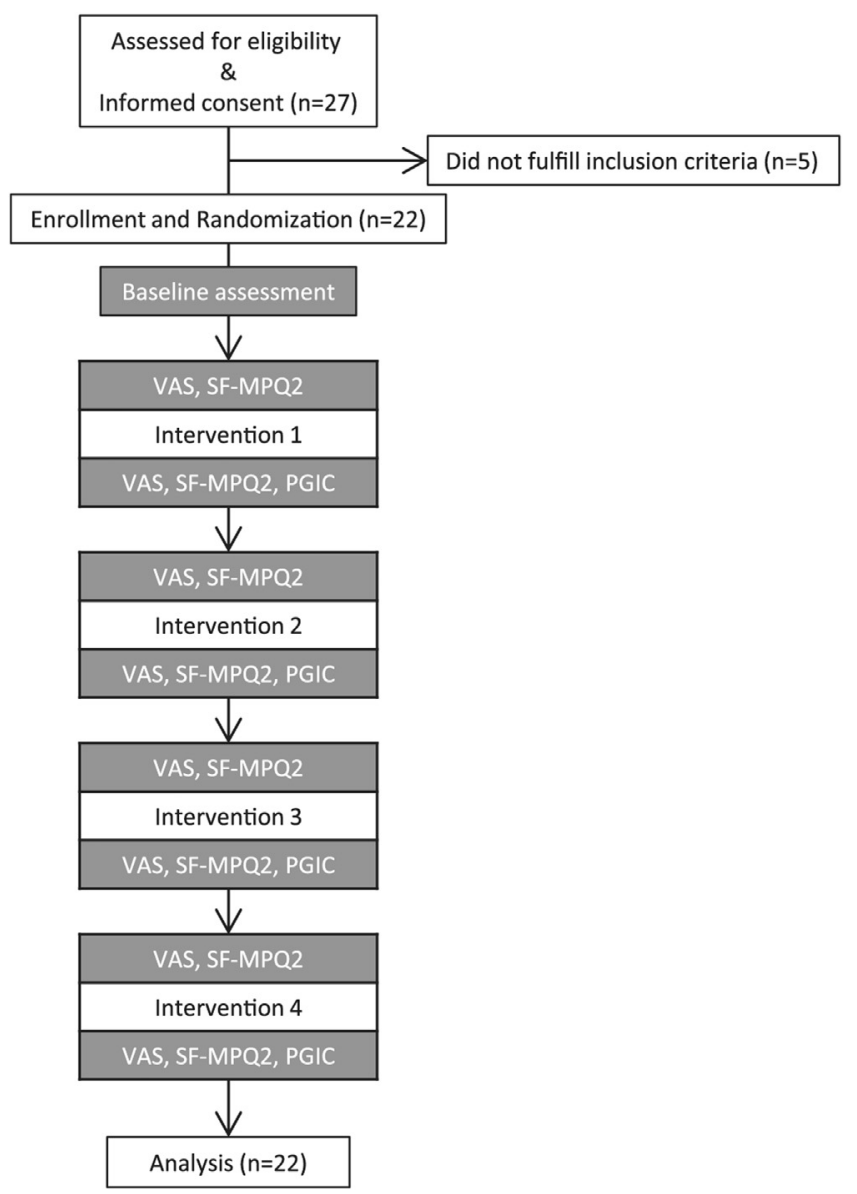

Figure 1. Study flowchart. Four single sessions of intervention were administered in random order. Pain scores were collected immediately before and after the intervention. PGIC (1: "very much improved"; 2: "much improved"; 3 "minimally improved"; 4: "no change"; 5: "minimally worse"; 6: "much improved"; and 7: "very much worse") was determined immediately after each intervention. A definition of interventions is in the text. VAS, visual analog scale: SF-MPQ2, Short-Form McGill Pain Questionnaire 2; PGIC, Patient Global Impression of Change. 
pain in the past, and 4) age 20 years or older. Exclusion criteria were inability to complete the written questionnaires, dementia (Mini-Mental State Examination [MMSE] score $\leq 23$ ], severe aphasia, higher brain dysfunction, major psychiatric disorder, suicidal ideation, pregnancy, history of epilepsy, or contraindication to TMS (eg, implanted cardiac pacemaker). ${ }^{31,32}$ Participants were requested to not discontinue or change their medications during the study period and to continue their routine analgesic medication regimen throughout the study.

\section{rTMS Protocol}

Active rTMS was performed using a stimulator (MagPro X100, MagVenture, Denmark) that induced biphasic magnetic pulses via a figure-8 coil (MC-B70, MagVenture). Sham-rTMS was delivered using a sham coil (MC-P-B70, MagVenture) that mimicked the active coil visually and audibly but delivered no significant magnetic stimulation.

During all sessions, the patients were seated in a comfortable reclining chair. The center of the coil was placed over the $\mathrm{M} 1$ hand area contralateral to the painful side. We decided to target the M1 hand area in this trial because it is easier to stimulate than the M1 face and foot areas. Before the first session, the stimulation site and resting motor threshold (RMT) were determined in most patients by recording motor evoked potentials (MEPs) in the first dorsal interosseous muscle. In the patient with phantom limb pain, the deltoid muscle was used instead. MEPs were recorded using Ag/ $\mathrm{AgCl}$ surface electrodes in a belly-tendon montage. Electromyogram signals were amplified with a $500-3000 \mathrm{~Hz}$ band pass filter and digitally sampled at $10 \mathrm{kHz}$ for storage in the Brainsight navigation system (Rogue Research Inc, Montreal, Canada). The stimulation site was set as the cortical site where a single TMS pulse elicited an MEP of maximal amplitude; this site was subsequently saved in the Brainsight system. RMT was defined as the minimal intensity required to induce at least five MEPs of $\geq 50 \mu \mathrm{V}$ in ten consecutive MEP recordings. ${ }^{21,33}$

In all treatment sessions, the stimulation intensity was set at $90 \%$ of the RMT. Patients received each of the following protocols in random order: 1 ) active $5-\mathrm{Hz}$ rTMS with 500 pulses (ten trains of 10 $\mathrm{sec}$, separated by an inter-train interval [ITI] of $50 \mathrm{sec}$ ); 2) active 10$\mathrm{Hz}$ rTMS with 500 pulses (ten trains of $5 \mathrm{sec}$, separated by an ITI of $25 \mathrm{sec}$ ); 3) active 10- $\mathrm{Hz}$ rTMS with 2000 pulses (40 trains of $5 \mathrm{sec}$, separated by an ITI of $25 \mathrm{sec}$ ); and 4) sham stimulation (same parameters as the active $5-\mathrm{Hz}$ rTMS treatment). The coil was positioned tangentially to the scalp and perpendicular to the central sulcus. The TMS coil was oriented in the anterior-posterior direction. $^{13,25,26,34}$ The Brainsight navigation system was used to monitor accurate positioning and direction of the coil as well as the position of the patient's head throughout each individual session and across sessions. ${ }^{19}$ T1-weighted images were obtained using these parameters: field of view, $256 \times 256 \times 176 \mathrm{~mm}$; voxel size, $1 \times 1 \times 1 \mathrm{~mm}$; TR, $8.164 \mathrm{msec}$; TE, $3.184 \mathrm{msec}$; Tl, $400 \mathrm{msec}$; and flip angle, $11^{\circ}$. This $\mathrm{rTMS}$ protocol was in compliance with the guidelines for safe use of rTMS. ${ }^{31,32}$

\section{Clinical Assessments}

The following self-assessment scoring systems were used prior to the first rTMS session to determine the patients' baseline characteristics: Beck Depression Inventory-II (BDI-II), Hospital Anxiety and Depression Scale (HADS), Pain Disability Assessment Scale (PDAS), Pain Catastrophizing Scale (PCS), and 5-level version of EuroQol
5-Dimension (EQ-5D-5L). VAS pain intensity and the Japanese version of the Short-Form McGill Pain Questionnaire 2 (SF-MPQ2) (scale range: $0-220$ ) were assessed at baseline and immediately before and after each intervention. Patient Global Impression of Change $(P G I C)$, which is a 7-point scale ranging from "very much improved" ${ }^{1}$ to "very much worse", 7 was recorded immediately after each intervention. $^{35}$ To assess the effectiveness of blinding, patients were asked at the end of the study whether they recognized the sham treatment. All assessment items were contained on unified forms, which were completed by each patient. Figure 1 represents the flowchart of the study.

\section{Statistical Analysis}

VAS and SF-MPQ2 decreases for each session were calculated by subtracting the value immediately after the intervention from that immediately before the intervention. Statistical analyses of VAS decrease, SF-MPQ2 decrease, and PGIC were performed using a linear mixed-effects model (fixed effect: intervention, order, and interaction between intervention and order; random effect: patient). Additionally, we evaluated the influence of background factors (peripheral vs central neuropathic pain, severity of motor disturbance, and severity of sensory disturbance) by adding each background factor and interaction between intervention and background factor as fixed effects to the statistical model. Tukey multiple comparison tests were used to investigate differences in analgesic effects between each pair of interventions. To evaluate possible carry-over effects, one-way ANOVA was applied to the values during each intervention period. In all analyses, findings with a two-sided $p$ value $<0.05$ were considered statistically significant. JMP Pro version 14 (SAS Institute) was used for the statistical analyses.

\section{RESULTS}

We screened 27 patients with chronic pain for participation in the trial; five were excluded because they did not meet the diagnostic criteria $(n=4)$ or their baseline VAS pain intensity was less than $30 \mathrm{~mm}(n=1)$. A total of 22 patients (mean age: $59.8 \pm 12.1$ years; 11 males and 11 females) with neuropathic pain were thereby enrolled in this exploratory study. The etiologies of neuropathic pain were as follows: central poststroke pain, 15 patients; complex regional pain syndrome, 3 patients; peripheral nerve injury, 2 patients; spinal lesion, 1 patient; and root avulsion, 1 patient. All participants completed the four stimulation sessions in the assigned order and were included in the statistical analyses (Fig. 1). No lasting side effects, including convulsions, were observed. Two patients reported transient scalp pain following active rTMS. MEPs were recorded from the deltoid muscle in patients with phantom limb pain, and from the first dorsal interosseous muscle in the remaining patients. Seven of the 22 patients (32\%) correctly identified the sham stimulation session. Table 1 presents the patients' baseline demographic and clinical characteristics.

\section{Effects of rTMS on VAS, SF-MPQ2 Changes, and PGIC}

Mean (standard deviation) VAS pain intensity decreased from 58.4 (26.2) to 51.0 (30.1) at 5- $\mathrm{Hz}$ with 500 pulses, from 63.7 (25.2) to $59.2(27.9)$ at $10-\mathrm{Hz}$ with 500 pulses, from 66.0 (19.6) to 55.3 (27.7) at $10-\mathrm{Hz}$ with 2000 pulses, and from 62.5 (20.1) to 59.7 (24.1) with sham stimulation (Fig. 2a). Of the four conditions, 2000 pulses per 
Table 1. Patients' Characteristics at Baseline $(N=22)$.

\begin{tabular}{|c|c|}
\hline Age (y) & $59.8 \pm 12.1$ \\
\hline Sex (female, male) & 11,11 \\
\hline \multicolumn{2}{|l|}{ Origin of pain } \\
\hline Central post-stroke pain & 15 \\
\hline Complex regional pain syndrome & 3 \\
\hline Peripheral nerve injury & 2 \\
\hline Spinal lesion & 1 \\
\hline Root avulsion & 1 \\
\hline \multicolumn{2}{|l|}{ Treated painful region } \\
\hline Right, left & 9,13 \\
\hline Face, upper limb, lower limb & $2,10,10$ \\
\hline Pain duration (mo), median (interquartile range) & $49(29-64)$ \\
\hline VAS pain intensity (mm) & $72.5 \pm 11.3$ \\
\hline SF-MPQ2 & $85.4 \pm 38.8$ \\
\hline Motor disturbance* (Normal-mild, moderate-severe) & 13,9 \\
\hline Sensory disturbance (Normal-mild, moderate-severe) & 10,12 \\
\hline MMSE (0-30) & $28.7 \pm 1.6$ \\
\hline PDAS $(0-60)$ & $26.2 \pm 14.1$ \\
\hline EQ-5D-5L (0-1) & $0.583 \pm 0.19$ \\
\hline PCS $(0-52)$ & $27.0 \pm 10.7$ \\
\hline rumination (0-20) & $14.2 \pm 4.0$ \\
\hline helplessness (0-20) & $8.4 \pm 4.8$ \\
\hline magnification (0-12) & $4.5 \pm 3.2$ \\
\hline HADS anxiety $(0-21)$ & $5.7 \pm 4.2$ \\
\hline HADS depression (0-21) & $5.7 \pm 3.2$ \\
\hline BDI-II (0-63) & $11.3 \pm 9.2$ \\
\hline Current medication regime (\%) & $22(100 \%)$ \\
\hline Pregabalin & $14(63.6 \%)$ \\
\hline Duloxetine & $8(36.4 \%)$ \\
\hline Tramadol & $7(31.8 \%)$ \\
\hline Baclofen & $5(22.7 \%)$ \\
\hline Amitriptyline & $3(13.6 \%)$ \\
\hline NSAIDs & $3(13.6 \%)$ \\
\hline Acetaminophen & $2(9.1 \%)$ \\
\hline Clonazepam & $2(9.1 \%)$ \\
\hline Carbamazepine & $1(4.5 \%)$ \\
\hline Neurotropin & $1(4.5 \%)$ \\
\hline Tramadol and acetaminophen & $1(4.5 \%)$ \\
\hline Nortriptyline & $1(4.5 \%)$ \\
\hline
\end{tabular}

Data are mean \pm standard deviation or number, unless otherwise indicated. Numbers in parentheses after the scoring systems indicate the range of possible scores.

VAS, visual analog scale; SF-MPQ2, Short-Form McGill Pain Questionnaire 2; MMSE, Mini-Mental State Examination; PDAS, Pain Disability Assessment Scale; EQ-5D, EuroQol-5 Dimension; PCS, Pain Catastrophizing Scale; HADS, Hospital Anxiety and Depression Scale; BDI-II, Beck Depression Inventory Version 2.

*Normal to mild motor deficit was defined as muscle strength of grade 4 or more on the painful side, in accordance with Medical Research Council scores (22).

session at $10 \mathrm{~Hz}$ produced the best pain relief. Mean $(95 \% \mathrm{CI})$ VAS pain intensity decreases immediately after stimulation were $7.8 \mathrm{~mm}$ (2.8-12.9) for 5-Hz rTMS with 500 pulses, 4.3 (-0.7-9.3) for $10-\mathrm{Hz}$ rTMS with 500 pulses, $11.0(6.1-15.9)$ for $10-\mathrm{Hz}$ rTMS with 2000 pulses, and $2.8(-2.1-7.7)$ for sham stimulation. Mean $(95 \% \mathrm{Cl})$ VAS reduction rates were $18.4 \%$ (5.9-30.8), 13.9\% (1.6-26.1), 19.3\% (6.3-32.4), and $7.1 \%(-2.3-16.5)$, respectively. The linear mixedeffects model revealed a significant effect of intervention (ie, stimulation condition) on changes in VAS pain intensity $(F=3.3$, d.f. $=59.8 ; p=0.03$ ). Pain intensity was not affected by intervention order $(F=3.4$, d.f. $=66.1 ; p=0.07)$ or intervention interaction ( $F=1.4$, d.f. $=72.6 ; p=0.27$ ). Tukey multiple comparison test revealed that VAS pain intensity decreased significantly more with $10-\mathrm{Hz}$ rTMS with 2000 pulses than with sham intervention ( $p=0.03$ ) (Fig. 3a). There were no significant differences between the other stimulation conditions. Origin of pain, severity of motor disturbance, and severity of sensory disturbance did not influence the intervention effects $(p=0.99, p=0.78$, and $p=0.88$, respectively).

Mean (standard deviation) SF-MPQ2 decreased from 70.5 (53.6) to 56.0 (53.5) at 5- $\mathrm{Hz}$ with 500 pulses, from 75.6 (52.4) to 64.8 (51.8) at $10-\mathrm{Hz}$ with 500 pulses, from $73.2(51.2)$ to 53.9 (51.6) at $10-\mathrm{Hz}$ with 2000 pulses, and from 71.8 (48.5) to 63.1 (50.5) with sham stimulation (Fig. 2b). The short-term SF-MPQ2 results were similar to those for VAS pain intensity. Mean (95\% Cl) SF-MPQ2 decrease immediately after stimulation was 16.5 (9.4-23.5) for 5- Hz rTMS with 500 pulses, 10.4 (3.4-17.4) for 10-Hz rTMS with 500 pulses, 19.1 (12.2-26.0) for 10-Hz rTMS with 2000 pulses, and 8.4 (1.6-15.3) for sham stimulation. Mean $(95 \% \mathrm{Cl})$ SF-MPQ2 reduction rates were $29.1 \%$ (15.0-43.2), 17.8\% (6.7-28.9), 32.9\% (18.4-47.5), and $17.8 \%$ (5.9-29.7), respectively. The linear mixed-effects model revealed a significant effect of intervention on changes in SF-MPQ2 ( $F=4.4$, d.f. $=58.2 ; p=0.01)$. SF-MPQ2 was not affected by intervention $\operatorname{order}(F=1.6$, d.f. $=62.1 ; p=0.21)$ or intervention interaction $(F=$ 2.8 , d.f. $=66.7 ; p=0.049)$. Tukey multiple comparison test revealed that SF-MPQ2 decreased more with 10-Hz rTMS with 2000 pulses than with sham intervention ( $p=0.01$ ) (Fig. 3b). Origin of pain, severity of motor disturbance, and severity of sensory disturbance did not influence the intervention effects $(p=0.43, p=0.98$, and $p=0.84$, respectively). The type of simulation condition had no significant effect on PGIC (Fig. 3c). There was no detectable carryover effect for VAS, SF-MPQ2, or PGIC, as there were no significant differences in pretreatment values between sessions $(p=0.28,0.41$, and 0.44 , respectively).

\section{DISCUSSION}

In this study, we evaluated the analgesic effects of four different rTMS conditions to explore the optimal condition for treating neuropathic pain. Our findings showed that compared to sham, 10$\mathrm{Hz}$ rTMS with 2000 pulses produced a significant analgesic effect, whereas $5-\mathrm{Hz}$ or $10-\mathrm{Hz}$ rTMS with 500 pulses did not. These results suggest that high-dose rTMS (10-Hz and 2000 pulses) over M1 may be a recommended strategy for chronic pain.

We previously reported that $5-\mathrm{Hz}$ or $10-\mathrm{Hz}$ rTMS with 500 pulses improved neuropathic pain, when compared with sham or $1-\mathrm{Hz}$ rTMS. $^{14,19,21,36}$ However, mean short-term VAS reduction rates after five or ten sessions of rTMS with these stimulation conditions were only $6.3-6.5 \%$ in our multicenter randomized controlled trials. ${ }^{21}$ Moreover, our subsequent large rigorous trial failed to show positive results for the primary outcome: specifically, pain relief after 5 $\mathrm{Hz}$ rTMS with 500 pulses was modest and transient. ${ }^{23}$ Conversely, most other studies adopted higher doses of rTMS, such as 2000-3000 pulses/session and 10-20 Hz, and reported better outcomes. $^{22,25-28}$ In a recent systematic review, the analgesic effects of high-frequency rTMS for chronic pain were evaluated by meta-analysis of data from 25 studies with low or unclear risk of bias. ${ }^{22}$ Of these studies, 6 used less than 1000 pulses per session, 14 used 1000 to 1999 pulses per session, and 5 used 2000 pulses per session or more; that is, approximately $80 \%$ of the studies involved 
a

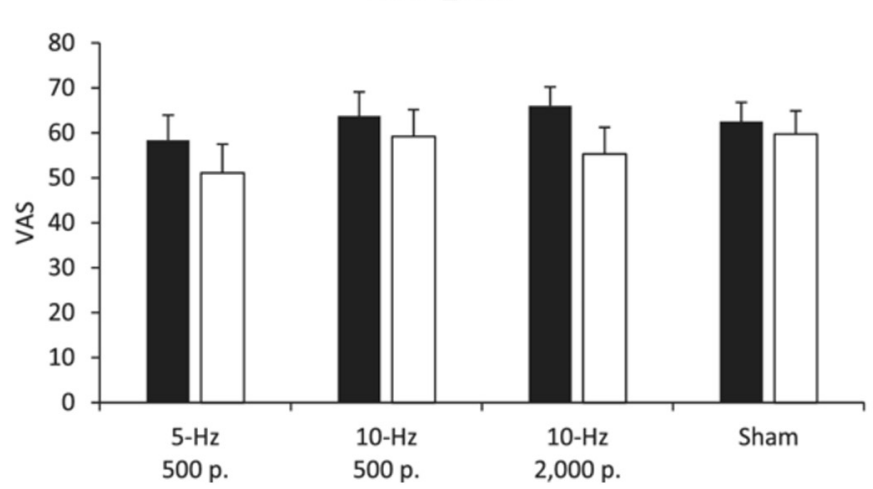

b

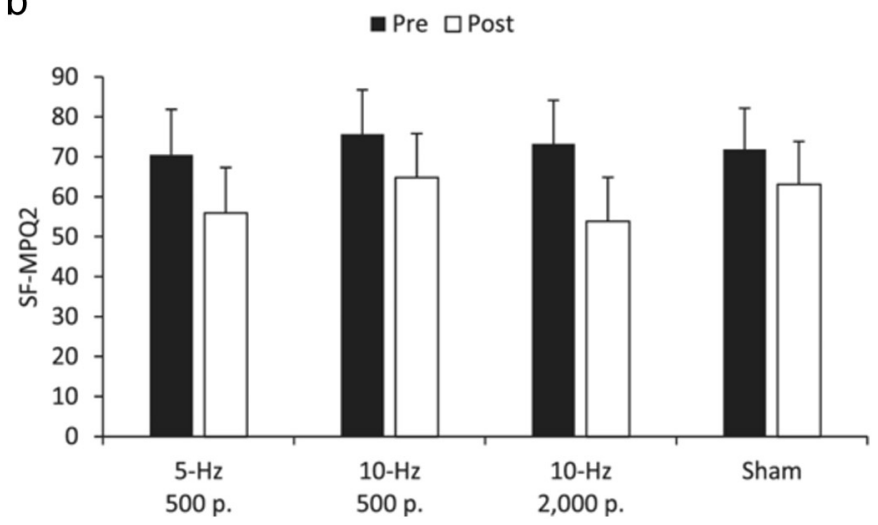

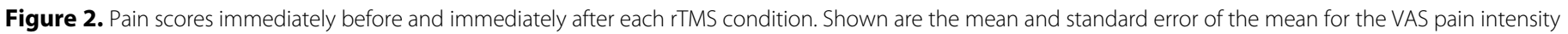

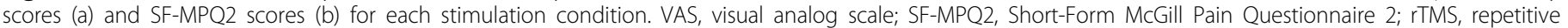
transcranial magnetic stimulation; p., pulses per session.

the use of more than 1000 pulses per session. In addition, a few studies compared the efficacy of $5-\mathrm{Hz}$ vs $10-\mathrm{Hz}$ rTMS. ${ }^{14,37}$ We previously reported that $10-\mathrm{Hz}$ rTMS with 500 pulses tended to produce better pain relief than $5-\mathrm{Hz}$ rTMS with 500 pulses, although the difference in analgesic effects between frequencies did not reach statistical significance. ${ }^{14}$ Similarly, Pei et al found that, after multiple sessions, the analgesic effect was significantly larger with $10-\mathrm{Hz}$ rTMS with 1500 pulses than with $5-\mathrm{Hz}$ rTMS with 1500 pulses per session or sham stimulation. ${ }^{37}$ In the present study, the analgesic effects of 5 - or $10-\mathrm{Hz}$ rTMS with 500 pulses were not significantly different from those of sham stimulation, whereas pain relief was significantly greater with $10-\mathrm{Hz}$ rTMS with 2000 pulses than with sham.

In recent years, higher rTMS stimulation frequencies and total number of pulses have been used to improve efficacy when treating chronic pain. ${ }^{10,22}$ However, we cannot conclude that a higher frequency, such as $10-\mathrm{Hz}$ to $20-\mathrm{Hz}$, is more suitable for treating neuropathic pain because there was no significant difference between the effects of $5-\mathrm{Hz}$ and $10-\mathrm{Hz}$ rTMS with 500 pulses/ session, and we did not test $20-\mathrm{Hz}$ rTMS. Moreover, the stimulation duration differed between our four intervention conditions. Session durations for $5-\mathrm{Hz}$ with 500 pulses, $10-\mathrm{Hz}$ with 500 pulses, and 10$\mathrm{Hz}$ with 2000 pulses, and sham $(5-\mathrm{Hz}$ with 500 pulses) were approximately $10,5,20$, and $10 \mathrm{~min}$, respectively. Pain relief is known to correlate with the duration of intervention, and the analgesic effects of rTMS may also depend on stimulation duration and/or number of pulses. It is likely that a higher-dose of rTMS, especially with a larger number of pulses, leads to greater pain relief effects.

Other parameters in addition to stimulation frequency and number of pulses should be considered when optimal stimulation conditions are discussed. The optimal stimulation intensity and site of stimulation within $\mathrm{M} 1$ remain controversial. In previous studies, the stimulation intensity varied from $80 \%$ to $120 \%$ RMT for rTMS treatment of intractable chronic pain. ${ }^{22}$ Suprathreshold rTMS of M1 and stimulation at frequencies greater than $10-\mathrm{Hz}$ appear to increase the risk of seizures in patients with stroke. This may be why most studies have used subthreshold intensities (ie, $80 \%$ or $90 \%$ RMT). Regarding the optimal stimulation site, there are two strategies for determining the stimulation site within M1. Some a

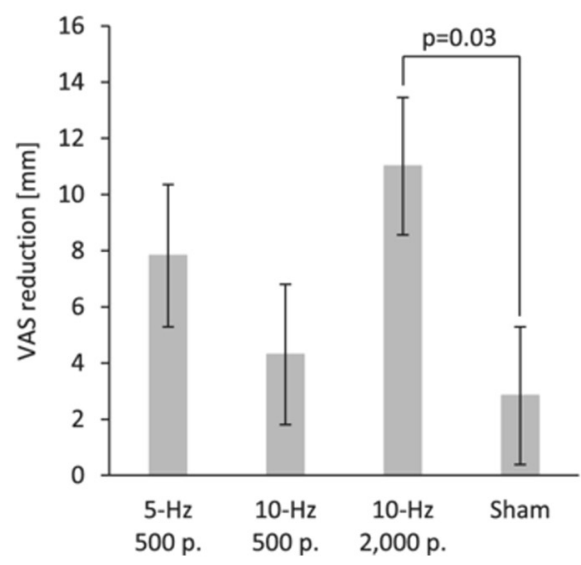

b

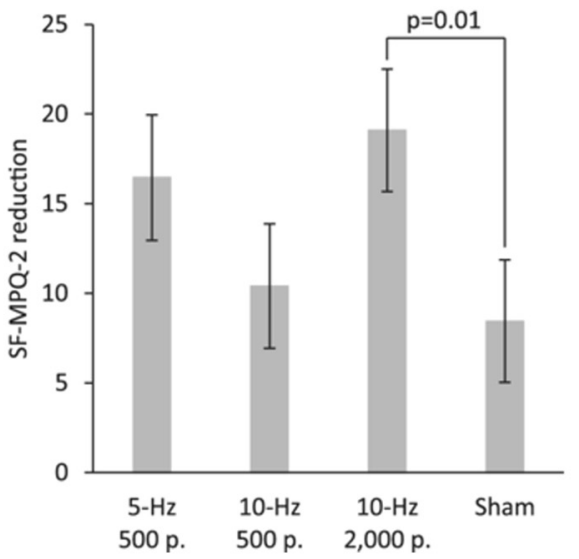

C

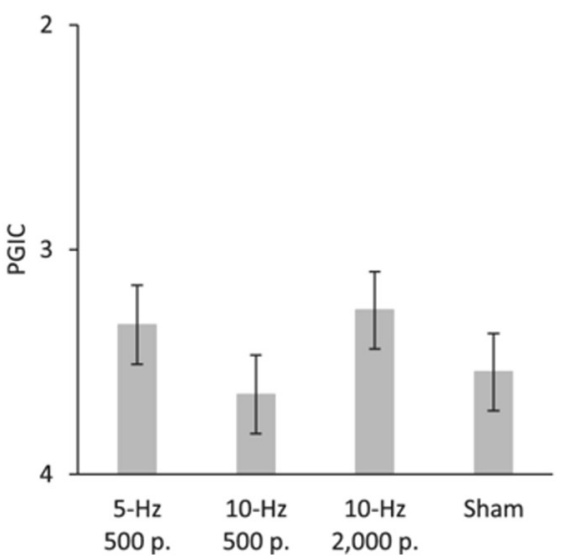

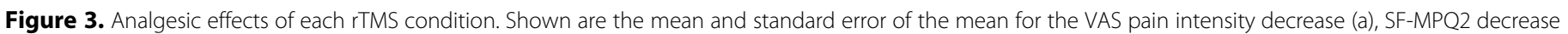

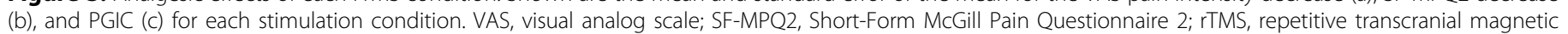
stimulation; p., pulses per session. 
studies stimulated the $\mathrm{M} 1$ hand area regardless of the location of the pain, ${ }^{38,39}$ whereas other studies stimulated the $M 1$ area somatotopically corresponding to the painful region. ${ }^{21,23,40}$ Previous reports indicated that the site of stimulation for rTMS was unrelated to analgesic effects, ${ }^{34,38}$ with several studies reporting pain relief in the foot or face after rTMS of the M1 hand area. ${ }^{26,34,38,39}$ In a previous study targeting premotor cortex/ dorsolateral prefrontal cortex areas other than M1, rTMS did not improve pain. ${ }^{41}$ According to a recent systematic review and society guidelines, most rTMS studies have involved stimulation of M1, for which there is weak to definite evidence of effectiveness. ${ }^{9,22,42}$ Therefore, stimulation was performed over the $M 1$ hand area contralateral to the neuropathic pain region in the present study. Carry-over effects should also be considered because one session may affect the outcomes of a subsequent session. However, there is no clear consensus regarding the interval between sessions necessary to prevent carry-over effects from previous rTMS sessions. Some studies have reported that analgesic effects of rTMS persist for up to six weeks, ${ }^{16,17}$ whereas other studies reported no significant carry-over effect over two weeks. ${ }^{21,43}$ In the current study, we separated each rTMS session from the preceding session by at least two weeks and detected no significant carry-over effects. However, the possibility of carry-over cannot be completely eliminated. Further studies with a large number of patients with neuropathic pain are required to conclusively establish the optimal stimulation intensity and M1 target area.

The analgesic effects of rTMS over M1 arise from modification of pain perception. ${ }^{44,45}$ The mechanism underlying pain relief in response to direct motor cortex stimulation (MCS) has been investigated in positron emission tomography (PET) activation studies. ${ }^{46}$ MCS appears to modulate several brain areas involved in pain perception through activation of several pain-related structures, including the thalamus, anterior cingulate cortex, and upper brainstem regions. ${ }^{47}$ The mechanism for analgesia in response to high-frequency rTMS may be similar to that of MCS. Moreover, there is a general consensus that low $(\leq 1 \mathrm{~Hz})$ and high $(\geq 5 \mathrm{~Hz})$ rTMS frequencies exert different effects upon cortical excitability: low-frequency rTMS inhibits cortical excitability, whereas high-frequency rTMS facilitates it. ${ }^{48-51}$ A PET study showed that rTMS at 20 $\mathrm{Hz}$ significantly increased global blood flow, whereas rTMS at $1 \mathrm{~Hz}$ did not. ${ }^{50}$ Several brain regions associated with pain perception may be activated by subthreshold high-frequency rTMS over M1 and thereby reduce deafferentation pain in a comprehensive manner.

Because of the lack of a concrete diagnostic tool for neuropathic pain, the IASP and European Federation of Neurological Societies (EFNS) suggested a grading system for neuropathic pain. ${ }^{2,3,30}$ In the present study, patients with neuropathic pain were diagnosed by specialist clinicians based on this grading system. ${ }^{1,30}$ Finnerup et al found that $30 \%$ of clinical studies published in 2014 used this system to classify or include patients with neuropathic pain, and while other studies used various questionnaires, such as the neuropathic pain questionnaire, the Douleur Neuropathique en 4 questions, and the painDETECT, as screening tools. ${ }^{29}$ These questionnaires rely on self-reporting by patients, which may limit their diagnostic accuracy. The EFNS states that the main usefulness of screening tools is to allow the identification of patients with potential neuropathic pain by nonspecialist clinicians and that $10-20 \%$ of patients with neuropathic pain cannot be identified using these questionnaires. ${ }^{3}$ Thus, although the reliability of a diagnosis of neuropathic pain by specialist clinicians can be enhanced by the use of a screening tool or grading system for neuropathic pain, the diagnosis can be difficult and should be interpreted with caution.

Several limitations of our study deserve consideration. First, the current study was only single (patient- and assessor-) blinded. The patients were not informed of the nature of each rTMS session and they self-assessed their pain-related scores, but the rTMS operator was aware of the stimulation conditions. The sensations caused by different methods, especially between active and sham coils, were different and may have theoretically influenced the results. However, the rate of correct answers for guessing the sham intervention indicate acceptable blinding. Second, we conducted only a single session for each condition to explore the optimal stimulation parameters for rTMS. Our findings showed that $10-\mathrm{Hz}$ rTMS with 2000 pulses produced short-term pain relief, but these effects do not necessarily represent clinically meaningful changes because patients with chronic pain require analgesia over a prolonged period of time. Moreover, the superiority of single $10-\mathrm{Hz}$ rTMS with 2000 pulses appeared to be weak, since its effects were not statistically different from the effects of other active stimulation parameters in the present study. This may be due to an insufficient number of sessions. Several studies investigating the effects of repeated rTMS sessions over several weeks or months have reported longer-term analgesic efficacies. ${ }^{16-18,37,52}$ Although most of these studies were open label and contained no control group, Quesada and colleagues recently reported the results of a randomized, double-blind, sham-controlled crossover trial. ${ }^{18}$ In that study, long-term rTMS was shown to have beneficial effects. Based on these results, future trials should explore the long-term benefits of rTMS. Another limitation was that we conducted rTMS for various types of neuropathic pain, including central poststroke pain, peripheral nerve injury, and others. Previous reviews suggested that rTMS at M1 contralateral to the side of pain is more effective for attenuating central neuropathic pain than peripheral neuropathic pain..$^{8-10}$ However, it is unclear whether differences in the origin of neuropathic pain affect the analgesic effects of rTMS because our previous reports showed that the analgesic effects of rTMS were better in patients with noncerebral lesions than in those with cerebral lesions ${ }^{14}$ and did not differ between patients with central vs peripheral neuropathic pain. ${ }^{21,23}$ In this way, we analyzed the influence of different origins of pain (peripheral vs central) and different degrees of motor and sensory disturbances on the effectiveness of rTMS and found that these factors did not affect rTMS effectiveness. However, because of the small number of subjects in this study, these findings should be interpreted with caution and warrant further evaluation in a future study. Another limitation was that we evaluated the analgesic effects of rTMS immediately before and after each intervention. A few previous studies have reported that beneficial effects of rTMS do not appear until a few days after the procedure. ${ }^{43,53}$ However, our previous studies evaluating pain scores over time in minute, hour, and day units revealed that maximum pain relief occurred immediately after rTMS, ${ }^{14,19,20}$ which is why we chose to evaluate the pain relieving effects of rTMS immediately before and after each intervention in the current study. Admittedly, pain relief immediately after intervention is less important than long-term analgesic effects, so longterm pain relief should be assessed in future, confirmatory trials. Lastly, the number of patients in the current study was not sufficiently large to reach definitive conclusions. Crossover studies with multiple arms often require a large number of patients. Although the relatively small number of patients increased the risk of bias, 
the small size of this study allowed us to obtain preliminary information over a relatively short period of time, permitting us to quickly determine appropriate stimulation parameters for evaluation in a future large randomized controlled trial. Of note, the number of patients was also insufficient to perform multivariate analysis to more rigorously examine the effects of such variables as the number of stimuli, duration, and frequency. Therefore, the findings of the present study should be interpreted with caution and considered preliminary, with a large-scale study required in the future. Based on recent reports and expert panel recommendations, we suggest that a future long-term study consist of five to ten induction sessions over one to two weeks at 10-Hz rTMS with 2000 pulses/session, followed by five to ten maintenance sessions once per week. ${ }^{10,54}$

\section{CONCLUSIONS}

The present study investigated the effects of variations in stimulation frequency and number of pulses per session on the analgesic benefits of rTMS for neuropathic pain. Of the conditions tested, $10-\mathrm{Hz}$ rTMS with 2000 pulses per session was the most effective. Several methodological issues require further investigation, including the optimal target site and the influence of session duration and pulse number on the analgesic effects. A larger-scale study is necessary to reach definitive conclusions.

\section{Authorship Statements}

Koichi Hosomi, Nobuhiko Mori, and Youichi Saitoh contributed to the trial design. Youichi Saitoh, Satoru Oshino, and Haruhiko Kishima supervised conduct of the trial. Experiments and data collection were conducted by Nobuhiko Mori and Koichi Hosomi. All authors contributed to the data interpretation. Koichi Hosomi and Nobuhiko Mori wrote the manuscript, Youichi Saitoh edited it, and all other authors reviewed it. Koichi Hosomi was responsible for the statistical analysis. All authors approved the final manuscript.

\section{How to Cite This Article}

Mori N., Hosomi K., Nishi A., Oshino S., Kishima $H_{\text {., }}$ Saitoh Y. 2022. Analgesic Effects of Repetitive Transcranial Magnetic Stimulation at Different Stimulus Parameters for Neuropathic Pain: A Randomized Study.

Neuromodulation 2022; 25: 520-527.

\section{REFERENCES}

1. Treede RD, Jensen TS, Campbell JN, et al. Neuropathic pain: redefinition and a grading system for clinical and research purposes. Neurology. 2008;70:1630-1635.

2. Cruccu G, Aziz TZ, Garcia-Larrea L, et al. EFNS guidelines on neurostimulation therapy for neuropathic pain. Eur J Neurol. 2007;14:952-970.

3. Cruccu G, Sommer $C$, Anand P, et al. EFNS guidelines on neuropathic pain assessment: revised 2009. Eur J Neurol, 2010;17:1010-1018.

4. van Hecke O, Austin SK, Khan RA, Smith BH, Torrance N. Neuropathic pain in the general population: a systematic review of epidemiological studies. Pain. 2014;155:654-662.

5. Inoue S, Taguchi T, Yamashita T, Nakamura M, Ushida T. The prevalence and impact of chronic neuropathic pain on daily and social life: a nationwide study in a Japanese population. Eur J Pain. 2017:21:727-737.
6. Smith $\mathrm{BH}$, Torrance N, Bennett MI, Lee AJ. Health and quality of life associated with chronic pain of predominantly neuropathic origin in the community. Clin J Pain 2007;23:143-149.

7. Inoue S, Kobayashi F, Nishihara M, et al. Chronic pain in the Japanese communityprevalence, characteristics and impact on quality of life. PLoS One. 2015;10: e0129262.

8. Bates D, Schultheis BC, Hanes $M C$, et al. A comprehensive algorithm for management of neuropathic pain. Pain Med. 2019;20:S2-S12.

9. Lefaucheur JP, Andre-Obadia N, Antal A, et al. Evidence-based guidelines on the therapeutic use of repetitive transcranial magnetic stimulation (rTMS). Clin Neurophysiol. 2014;125:2150-2206.

10. Leung A, Shirvalkar $P$, Chen R, et al. Transcranial magnetic stimulation for pain headache, and comorbid depression: INS-NANS expert consensus panel review and recommendation. Neuromodulation. 2020:23:267-290.

11. Leung $A$, Donohue $M, X u R$, et al. rTMS for suppressing neuropathic pain: a metaanalysis. J Pain. 2009;10:1205-1216.

12. Lefaucheur JP, Drouot $X$, Keravel $Y$, Nguyen JP. Pain relief induced by repetitive transcranial magnetic stimulation of precentral cortex. Neuroreport. 2001;12:29632965.

13. Andre-Obadia N, Peyron R, Mertens P, Mauguiere F, Laurent B, Garcia-Larrea L. Transcranial magnetic stimulation for pain control. Double-blind study of different frequencies against placebo, and correlation with motor cortex stimulation efficacy. Clin Neurophysiol. 2006;117:1536-1544.

14. Saitoh $Y$, Hirayama A, Kishima $H$, et al. Reduction of intractable deafferentation pain due to spinal cord or peripheral lesion by high-frequency repetitive trans cranial magnetic stimulation of the primary motor cortex. I Neurosurg. 2007:107:555-559.

15. Lefaucheur JP, Drouot X, Menard-Lefaucheur I, Keravel Y, Nguyen JP. Motor cortex rTMS in chronic neuropathic pain: pain relief is associated with thermal sensory perception improvement. J Neurol Neurosurg Psychiatry. 2008;79:1044-1049.

16. Pommier B, Creac'h C, Beauvieux V, Nuti C, Vassal F, Peyron R. Robot-guided neuronavigated rTMS as an alternative therapy for central (neuropathic) pain: clinical experience and long-term follow-up. Eur J Pain. 2016:20:907-916.

17. Quesada C, Pommier B, Fauchon C, et al. Robot-guided neuronavigated repetitive transcranial magnetic stimulation (rTMS) in central neuropathic pain. Arch Phys Med Rehabil. 2018;99:2203-2215.

18. Quesada C, Pommier B, Fauchon C, et al. New procedure of high-frequency repetitive transcranial magnetic stimulation for central neuropathic pain: a placebo-controlled randomized crossover study. Pain. 2020;161:718-728.

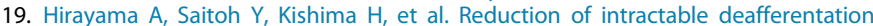
pain by navigation-guided repetitive transcranial magnetic stimulation of the primary motor cortex. Pain. 2006;122:22-27.

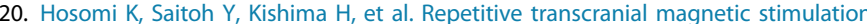
for neuropathic pain. Funct Neurosurg. 2008;47:135-140.

21. Hosomi K, Shimokawa T, Ikoma K, et al. Daily repetitive transcranial magnetic stimulation of primary motor cortex for neuropathic pain: a randomized, multicenter, double-blind, crossover, sham-controlled trial. Pain. 2013:154:1065-1072.

22. O'Connell NE, Marston L, Spencer S, DeSouza LH, Wand BM. Non-invasive brain stimulation techniques for chronic pain. Cochrane Database Syst Rev. 2018:4:CD008208.

23. Hosomi K, Sugiyama K, Nakamura Y, et al. A randomized controlled trial of 5 daily sessions and continuous trial of 4 weekly sessions of repetitive transcranial magnetic stimulation for neuropathic pain. Pain. 2020;161:351-360.

24. Hosomi K, Saitoh Y, Kishima $H$, et al. Non-invasive central nervous system stimulation for neurological diseases repetitive transcranial magnetic stimulation [in Japanese]. Funct Neurosurgery. 2010:49:2-3.

25. Attal N, Ayache SS, Ciampi De Andrade D, et al. Repetitive transcranial magnetic stimulation and transcranial direct-current stimulation in neuropathic pain due to radiculopathy: a randomized sham-controlled comparative study. Pain. 2016:157:1224-1231.

26. Ayache SS, Ahdab R, Chalah MA, et al. Analgesic effects of navigated motor cortex rTMS in patients with chronic neuropathic pain. Eur J Pain. 2016;20: $1413-1422$.

27. Khedr EM, Kotb HI, Mostafa MG, et al. Repetitive transcranial magnetic stimulation in neuropathic pain secondary to malignancy: a randomized clinical trial. Eur Pain. 2015:19:519-527.

28. Nurmikko $T$, Maclver $K$, Bresnahan R, Hird E, Nelson A, Sacco P. Motor cortex reorganization and repetitive transcranial magnetic stimulation for pain-a meth odological study. Neuromodulation. 2016;19:669-678.

29. Finnerup NB, Haroutounian $S$, Kamerman $P$, et al. Neuropathic pain: an updated grading system for research and clinical practice. Pain. 2016;157:1599-1606.

30. Loeser JD, Treede RD. The Kyoto protocol of IASP basic pain terminology. Pain. 2008:137:473-477.

31. Wassermann EM. Risk and safety of repetitive transcranial magnetic stimulation: report and suggested guidelines from the international workshop on the safety of repetitive transcranial magnetic stimulation, June 5-7, 1996. Electroencephalogr Clin Neurophysiol. 1998;108:1-16.

32. Rossi S, Hallett M, Rossini PM, Pascual-Leone A, Safety of TMSCG. Safety, ethical considerations, and application guidelines for the use of transcranial magnetic stimulation in clinical practice and research. Clin Neurophysiol. 2009;120:20082039.

33. Rossini PM, Barker AT, Berardelli A, et al. Non-invasive electrical and magnetic stimulation of the brain, spinal cord and roots: basic principles and procedures for 
routine clinical application. Report of an IFCN committee. Electroencephalogr Clin Neurophysiol. 1994;91:79-92.

34. Andre-Obadia N, Magnin M, Simon E, Garcia-Larrea L. Somatotopic effects of rTMS in neuropathic pain? A comparison between stimulation over hand and face motor areas. Eur J Pain. 2018;22:707-715.

35. Dworkin RH, Turk DC, Wyrwich KW, et al. Interpreting the clinical importance of treatment outcomes in chronic pain clinical trials: IMMPACT recommendations. J Pain. 2008:9:105-121.

36. Shimizu T, Hosomi K, Maruo T, et al. Efficacy of deep rTMS for neuropathic pain in the lower limb: a randomized, double-blind crossover trial of an $\mathrm{H}$-coil and figure-8 coil. J Neurosurg. 2017;127:1172-1180.

37. Pei Q, Wu B, Tang Y, et al. Repetitive transcranial magnetic stimulation at different frequencies for postherpetic neuralgia: a double-blind, sham-controlled, randomized trial. Pain Physician. 2019;22:E303-E313.

38. Jette F, Cote I, Meziane HB, Mercier C. Effect of single-session repetitive transcranial magnetic stimulation applied over the hand versus leg motor area on pain after spinal cord injury. Neurorehabil Neural Repair. 2013;27:636-643.

39. Lefaucheur JP, Hatem S, Nineb A, et al. Somatotopic organization of the analgesic effects of motor cortex rTMS in neuropathic pain. Neurology. 2006;67: 1998-2004.

40. Saitoh Y, Maruo T, Yokoe M, Matsuzaki T, Sekino M. Electrical or repetitive transcranial magnetic stimulation of primary motor cortex for intractable neuropathic pain. Conf Proc IEEE Eng Med Biol Soc. 2013;2013:6163-6166.

41. de Oliveira RA, de Andrade DC, Mendonca M, et al. Repetitive transcranial magnetic stimulation of the left premotor/dorsolateral prefrontal cortex does not have analgesic effect on central poststroke pain. J Pain. 2014;15:1271-1281.

42. Cruccu G, Garcia-Larrea L, Hansson P, et al. EAN guidelines on central neurostimulation therapy in chronic pain conditions. Eur J Neurol. 2016;23: 1489-1499.

43. Andre-Obadia N, Mertens P, Gueguen A, Peyron R, Garcia-Larrea L. Pain relief by rTMS: differential effect of current flow but no specific action on pain subtypes. Neurology. 2008;71:833-840.
44. Pleger B, Janssen F, Schwenkreis P, Völker B, Maier C, Tegenthoff M. Repetitive transcranial magnetic stimulation of the motor cortex attenuates pain perception in complex regional pain syndrome type I. Neurosci Lett. 2004;356:87-90.

45. Hosomi K, Seymour B, Saitoh Y. Modulating the pain network-neurostimulation for central poststroke pain. Nat Rev Neurol. 2015;11:290-299.

46. Kishima H, Saitoh Y, Osaki Y, et al. Motor cortex stimulation in patients with deafferentation pain: activation of the posterior insula and thalamus. J Neurosurg. 2007;107:43-48.

47. Garcia-Larrea L, Peyron R, Mertens P, et al. Electrical stimulation of motor cortex for pain control: a combined PET-scan and electrophysiological study. Pain 1999;83:259-273.

48. Fitzgerald PB, Fountain S, Daskalakis ZJ. A comprehensive review of the effects of rTMS on motor cortical excitability and inhibition. Clin Neurophysiol. 2006:117:2584-2596.

49. Kimbrell TA, Little JT, Dunn RT, et al. Frequency dependence of antidepressant response to left prefrontal repetitive transcranial magnetic stimulation (rTMS) as a function of baseline cerebral glucose metabolism. Biol Psychiatry. 1999;46:16031613.

50. Speer AM, Kimbrell TA, Wassermann EM, et al. Opposite effects of high and low frequency rTMS on regional brain activity in depressed patients. Biol Psychiatry. 2000;48:1133-1141.

51. Halawa I, Goldental A, Shirota Y, Kanter I, Paulus W. Less might be more: conduction failure as a factor possibly limiting the efficacy of higher frequencies in rTMS protocols. Front Neurosci. 2018;12:1-12.

52. Kobayashi M, Fujimaki T, Mihara B, Ohira T. Repetitive transcranial magnetic stimulation once a week induces sustainable long-term relief of central poststroke pain. Neuromodulation. 2015;18:249-254.

53. Lefaucheur JP, Drouot X, Nguyen JP. Interventional neurophysiology for pain control: duration of pain relief following repetitive transcranial magnetic stimulation of the motor cortex. Neurophysiol Clin. 2001:31:247-252.

54. Lefaucheur JP, Nguyen JP. A practical algorithm for using rTMS to treat patients with chronic pain. Neurophysiol Clin. 2019;49:301-307. 